\title{
Management of the Internal Nasal Valve
}

\author{
Li Shia Ng, Stephen Lo
}

\begin{abstract}
Nasal obstruction is one of the most common nose complaints. Internal nasal valve dysfunction is an important cause of nasal obstruction, particularly in patients who have a previous history of nasal trauma or reduction rhinoplasty. Correct assessment is crucial for accurate diagnosis and appropriate management planning. Various surgical and nonsurgical modalities for addressing the problem of internal nasal valve collapse are being reviewed in this paper. Each technique have their own advantages and disadvantage, and the choice depends on the underlying pathology, patient preference, availability of graft material. The rhinoplasty surgeon should have a thorough understanding of the available options as part of his/her armamentarium in dealing with internal nasal valve pathology.
\end{abstract}

Keywords: Internal nasal valve, Rhinoplasty.

How to cite this article: Shia Ng L, Lo S. Management of the Internal Nasal Valve. Int J Otorhinolaryngol Clin 2013;5(1):43-45.

Source of support: Nil

Conflict of interest: None declared

\section{INTRODUCTION}

Nasal valve collapse is a commonly overlooked cause of nasal obstruction. The nasal valve area can be divided into the external and internal valve. The internal nasal valve is the narrowest segment of the nasal airway and collapse of this region can give rise to significant increase in airway resistance.

\section{INTERNAL NASAL VALVE ANATOMY}

Lying about $1.3 \mathrm{~cm}$ deep to the nares, the internal nasal valve is bordered medially by the dorsal septum, laterally by the caudal margin of the upper lateral cartilage and inferiorly by the head of the inferior turbinate. In the Caucasian population, the internal nasal valve angle measures about 10 to 15 degrees and any further narrowing of this angle can result in collapse of the internal nasal valve area.

\section{PATHOPHYSIOLOGY OF INTERNAL NASAL VALVE COLLAPSE}

Any cause of narrowing around the internal nasal valve region, such as septal deviation, hypertrophied inferior turbinate and pyriform aperture stenosis can result in acceleration of airflow. According to Bernoulli's principle, this increase in speed occurs simultaneously with a decrease in intraluminal pressure. In addition, if the lateral nasal wall is weak, the entire lateral nasal wall collapses.

\section{DIAGNOSIS OF INTERNAL NASAL VALVE COLLAPSE}

A patient with previous nasal fracture or rhinoplasty can present with internal nasal valve collapse. As part of hump reduction, the fibrous attachment between the nasal septum and upper lateral cartilages may be disrupted and if this is not adequately repaired, the lateral nasal wall collapses. Another mechanism by which rhinoplasty may result in collapse is via osteotomies, with medial displacement of the nasal bone and upper lateral cartilage attached to it. Other causes include facial nerve paralysis, which results in a nonfunctional dilator naris muscle that plays a part in keeping the lateral nasal wall open. Aging can also weaken the fibroareolar tissues of the nasal sidewall and trauma may disrupt the patency of the nasal airway around the internal nasal valve.

Upper lateral cartilage collapse may be static or dynamic, unilateral or bilateral. Signs suggestive of internal nasal valve collapse are weak/malformed nasal cartilages, inspiratory collapse of lateral nasal wall or ptotic nasal tip. Anterior rhinoscopy can reveal deviation of the nasal septum, scarring and stenosis of the internal nasal valve region and inferior turbinate hypertrophy. Decongestion should be performed to determine if the nasal obstruction can be relieved medically. If so, the patients should pursue medical therapy prior to considering surgery. The Cottle maneuver is the traditional method for diagnosing nasal valve collapse. This maneuver involves retracting the cheek adjacent to the nasal alae superolaterally and in a positive test patients experience an audible or subjective improvement of symptoms. However, this test is not always reliable. The modified Cottle maneuver has been proposed to more accurately diagnose the area of collapse, by using an ear curette intranasally to support the lower and upper lateral cartilages separately and assessing if nasal patency is increased.

According to the American Academy of Otolaryngology (AAO) clinical consensus for nasal valve compromise, this condition is best evaluated with history and anterior rhinoscopy. Endoscopy and photography is useful but not routine and radiography is not useful. There is a lack of gold standard objective test for the diagnosis of internal nasal valve collapse. Acoustic rhinometry and rhinomanometry is not universally available or accepted and the current role of these investigations is mainly in research.

\section{MANAGEMENT OF INTERNAL NASAL VALVE COLLAPSE}

\section{Nonsurgical Management}

Patients who are unfit or unwilling to go for operation can be managed conservatively with external adhesive strips or 
internal nasal springs to hold the internal nasal valve open. The downside of management with these splints is that they can be uncomfortable to wear and some may find it cosmetically unacceptable.

Vaiman ${ }^{1}$ performed a small placebo-controlled study with 40 patients for the treatment of nasal valve collapse with high frequency transcutaneous and intranasal electrical stimulation of nasal muscles. Twelve out of 20 patients in the treatment group, compared to seven in the placebo group, had subjective improvement of symptoms. However, there was rapid decline of improvement after termination of treatment. The same author ${ }^{2}$ described biofeedback training using electromyography and a home exercise program for nasal muscle building. This therapy improved nasal obstruction subjectively in all patients, and in $86 \%$ of the patients, surgery was avoided. Nyte $^{3}$ described a technique of spreader graft injection using Radiesse (calcium hydroxyapatite microspheres in sodium carboxymethylcellulose carrier gel) for internal nasal valve collapse. This involves a three-point injection to widen the internal valve angle.

\section{Surgical Management}

Surgery remains the primary mode of treatment for nasal valve collapse (AAO consensus). However, the evidence is based mostly on uncontrolled studies (grade $\mathrm{C}$ evidence). Septoplasty and inferior turbinate reduction surgery are simple surgeries that can widen the internal nasal valve area, but do not directly address the problem of lateral wall collapse or narrow nasal valve angle. To address the above problems, the aims of surgery are (1) to widen the nasal valve angle, (2) to reposition upper lateral cartilages and (3) to add structural graft to support the lateral nasal wall.

The mainstay of treatment of internal nasal valve collapse is spreader graft placement, which has been popularized by Sheen. ${ }^{4}$ Spreader grafts widen the nasal valve angle, as well as strengthen the nasal valve area and esthetically, can restore width to an esthetically pinched, narrow nose. However, accidental damage to nasal mucosa during insertion of graft can result in scar formation and further narrowing of the nasal valve angle.

Variations of the spreader graft include the spreader flap, which was described by Gruber. ${ }^{5}$ This method does not require a graft and involves rolling up of the upper lateral cartilage upon itself. Unlike the spreader graft, which can be placed via a closed or open rhinoplasty approach, the spreader flap can only be performed via the open approach. Esthetically, dorsal height may be lost due to shortening of the upper lateral cartilage.
Other grafts include the upper lateral splay graft ${ }^{6}$ and butterfly graft, ${ }^{7}$ which are placed below and above the upper lateral cartilages respectively. These cartilage grafts are harvested from the concha and makes use of the natural curvature of the concha to spring the upper lateral cartilages open. The disadvantage of placement of these grafts is excessive widening of the nasal dorsum. Alar batten grafts ${ }^{8}$ are used to support flaccid lateral nasal wall but may result in alar fullness.

Suture techniques, such as the flaring sutures and lateral suspension sutures ${ }^{9}$ have been described to pull the upper lateral cartilages laterally and widen the nasal valve angle. Specific surgeries can also be performed to address the cause of narrowing, such as scar resection or Z-plasty ${ }^{10}$ for nostril stenosis.

There is a lack of studies describing treatment approaches in patients with severe refractory internal nasal valve collapse (both dynamic and static), who have failed to respond to conventional surgery. ${ }^{11}$ In our institution, we treat these cases using a double-Y titanium plate technique.

\section{HOW WE DO IT?}

Under local anesthesia, a $1.5 \mathrm{~cm}$ alar-facial groove incision is made. Deep to the incision, a pocket is created, superficial to the upper lateral cartilage medially, and to the anterior wall of the maxilla laterally. A $0.5 \mathrm{~mm}$ thick double-Y titanium plate (Matrix Midface, Synthes, Switzerland) is placed into the pocket, with the medial end stitched to the upper lateral cartilage and the lateral end secured to the anterior maxillary wall with two $4 \mathrm{~mm}$ screws. The procedure can be performed bilaterally if indicated and wounds were closed with 6/0 Prolene Polypropylene suture. The double-Y titanium plate fixation of the upper lateral cartilage is a minimally invasive procedure that can be done under local anesthesia. It provides a potentially permanent and sturdy anchor for the upper lateral cartilage, addressing the disadvantages of conventional procedures. However, the risks of implant extrusion and infection have to be considered. Nevertheless, this technique is an option for patients who do not improve after conventional surgery.

\section{CONCLUSION}

A variety of surgical techniques have been described to deal with nasal valve collapse. As far as we are aware, there are no randomized controlled trials on nasal valve surgery. Published literature in nasal valve surgery is frequented by technical description of surgical technique rather than evidence of long-term patient benefit. The pathophysiological role of the internal nasal valve in nasal 
obstruction has improved considerably, but the many surgical techniques described may reflect the uncertainty in the comparative benefit of each technique.

\section{REFERENCES}

1. Vaiman M, et al. Treatment of nasal valve collapse with transcutaneous and intranasal electric stimulation. Ear Nose Throat J 2004 Nov;83(11):757-62,764.

2. Vaiman M, et al. Biofeedback training of nasal muscles using internal and external surface electromyography of the nose. Am J Otolaryngol 2005 Sep-Oct;26(5):302-07.

3. Nyte C. Spreader graft injection with calcium hydroxyapatite: A nonsurgical technique for internal nasal valve collapse. Laryngoscope 2006 July;116:1291-92.

4. Sheen JH. Spreader graft: A method of reconstructing the roof of the middle nasal vault following rhinoplasty. Plast Reconstr Surg 1984 Feb;73(2):230-39.

5. Gruber RP. The spreader flap in primary rhinoplasty. Plast Reconstr Surg 2007 May;119(6):1903-10.

6. Guyuron B. Upper lateral splay graft. Plast Reconstr Surg 1998 Nov;102(6):2169-77.

7. Clark JM. The 'butterfly' graft in functional secondary rhinoplasty. Laryngoscope 2002 Nov;112(11):1917-25.
8. Toriumi DM, Josen J, Weinberger M, Tardy ME Jr. Use of alar batten grafts for correction of nasal valve collapse. Arch Otolaryngol Head Neck Surg 1997 Aug;123(8):802-08.

9. Paniello RC. Nasal valve suspension. An effective treatment for nasal valve collapse. Arch Otolaryngol Head Neck Surg 1996 Dec;122(12):1342-46.

10. Dutton JM, Neidich MJ. Intranasal Z-plasty for internal nasal valve collapse. Arch Facial Plast Surg 2008 May-Jun;10(3): 164-68.

11. Spielmann PM, White PS, Hussain SS. Surgical techniques for the treatment of nasal valve collapse: A systematic review. Laryngoscope 2009 Jul;119(7):1281-90.

\section{ABOUT THE AUTHORS}

\section{Li Shia Ng}

Associate Consultant, Department of Otorhinolaryngology, National University Hospital, Singapore

\section{Stephen Lo}

Adjunct Assistant Professor, Department of Otorhinolaryngology National University of Singapore School of Medicine, Chief of Facial Plastic Surgery, Tan Tock Seng Hospital, Singapore 